\title{
Síndrome da Encefalopatia Posterior Reversível (PRES) no ciclo gravídico puerperal: revisão bibliográfica
}

\author{
Posterior Reversible Encephalopathy Syndrome (PRES) in the gravidic-puerperal cycle: a \\ bibliographic review
}
Síndrome de Encefalopatía Reversible Posterior (PRES) en el ciclo de embarazo puerperal: una revisión bibliográfica

Márcia Cristina Taveira Pucci Green ${ }^{1 *}$, Felipe Pimenta Rodrigues², Karina Martins Milaré2.

\section{RESUMO}

Objetivo: Elencar as principais características fisiopatológicas, clínicas, diagnósticas e manejo terapêutico da Síndrome da Encefalopatia Posterior Reversível (PRES) em gestantes e puérperas. Métodos: Esta revisão da literatura usou a base de dados de pesquisa CAPES, com os descritores: "PRES", "Posterior Reversible Encephalopathy Syndrome", "Posterior Leukoencephalopathy Syndrome" e "Pregnancy", entre os anos de 2003 a 2019, resultando em 50 artigos que preencheram os critérios de inclusão. Resultados: Verificou-se que, os mecanismos fisiopatológicos que buscam explicar a ocorrência geral dessa síndrome, ainda não são claros. Os principais sinais clínicos identificados foram convulsões associadas a cefaleia e alterações visuais. O exame padrão-ouro é a ressonância magnética, preferencialmente em T1, T2, ADC e DWI, estando a restrição de difusão relacionada a um pior prognóstico. Feito o diagnóstico, é necessário o manejo terapêutico com estabilização da paciente, controles pressórico, controle de convulsões e afastar a causa base que desencadeou o processo da PRES. Considerações finais: A PRES é uma síndrome de início agudo, sendo importante o reconhecimento das suas características clínicas e diagnósticas. Por isso quando pensar em PRES? Sugerimos um recurso mnemônico para lembrar como diagnóstico diferencial. P: Pré-eclâmpsia e eclâmpsia; R: Restrição de difusão na Ressonância Magnética; E: Edema cerebral; S: Sintomas visuais.

Palavras-chave: Eclampsia, Síndrome da leucoencefalopatia posterior, Gravidez, Período pós-parto;

\begin{abstract}
Objective: List the main pathophysiological, clinical, diagnostic, and therapeutic management characteristics of Posterior Reversible Encephalopathy Syndrome (PRES) in pregnant and postpartum women. Methods: This literature review used the CAPES research database, with the descriptors: "PRES", "Posterior Reversible Encephalopathy Syndrome", "Posterior Leukoencephalopathy Syndrome" and "Pregnancy", between the years 2003 to 2019, resulting in 50 articles that met the inclusion criteria. Results: It was found that the pathophysiological mechanisms that seek to explain the general occurrence of this syndrome are not yet clear. The main clinical signs identified were seizures associated with headache and visual disturbances. The gold standard exam is magnetic resonance, preferably in T1, T2, ADC and DWI, with the diffusion restriction being related to a worse prognosis. Once the diagnosis has been made, therapeutic management with stabilization of the patient, blood pressure controls, seizure control and removing the underlying cause that triggered the PRES process is necessary. Final considerations: PRES is an acute onset syndrome, and it is important to recognize its clinical and diagnostic characteristics. So when to think about PRES? We suggest a mnemonic resource to remember as a differential diagnosis. P: Pre-eclampsia and eclampsia; R: Magnetic Resonance diffusion restriction; E: Cerebral edema; S: Visual symptoms.
\end{abstract}

Keywords: Eclampsia, Posterior leukoencephalopathy syndrome, Pregnancy, Postpartum period.

1 Santa Casa de Misericórdia de Franca, Franca - SP. *E-mail: puccicolpo105@gmail.com

2 Universidade de Franca (UNIFRAN), Franca - SP. 


\section{RESUMEN}

Objetivo: Enumerar las principales características clínicas, fisiopatológicos, diagnósticos y manejo terapéutico de síndrome de encefalopatía reversible posterior (PRES) en mujeres embarazadas y posparto. Métodos: Esta revisión de la literatura utilizó la base de datos de investigación CAPES, con los descriptores: "PRES", "Posterior Reversible Encephalopathy Syndrome", "Posterior Leukoencephalopathy Syndrome" e "Pregnancy", entre 2003 y 2019, lo que resultó en 50 artículos que cumplieron con los criterios de inclusión. Resultados: Se descubrió que los mecanismos fisiopatológicos que buscan explicar la aparición general de este síndrome aún no están claros. Los principales signos clínicos identificados fueron convulsiones asociadas con dolor de cabeza y cambios visuales. El examen estándar de oro es la resonancia magnética, preferiblemente en T1, T2, ADC y DWI, con la restricción de difusión relacionada con un peor pronóstico. Una vez realizado el diagnóstico, es necesario el manejo terapéutico con la estabilización del paciente, los controles de la presión arterial, el control de las convulsiones y la eliminación de la causa subyacente que desencadenó el proceso PRES. Consideraciones finales: La PRES es un síndrome de inicio agudo y es importante reconocer sus características clínicas y diagnósticas. Entonces, ¿cuándo pensar en PRES? Sugerimos un recurso mnemotécnico para recordar como diagnóstico diferencial. P: preeclampsia y eclampsia; R: restricción de difusión por resonancia magnética; E: edema cerebral; S: síntomas visuales.

Palabras clave: Eclampsia, Síndrome de leucoencefalopatía posterior, Embarazo, Periodo posparto.

\section{INTRODUÇÃO}

A Síndrome da Encefalopatia Posterior Reversível (PRES), também conhecida como Síndrome da Leucoencefalopatia Posterior, foi inicialmente descrita por Hinchey J, et al. (1996) em estudo retrospectivo de 15 casos. É definida como condição clínico-radiológica associada a causa-base onde o paciente pode apresentar atividade convulsiva, comprometimento do nível de consciência, cefaleia, anormalidades visuais, náusea e ou vômito e sinais neurológicos focais. As anormalidades nas imagens cerebrais são frequentemente simétricas e predominam na substância branca posterior, tipicamente reversíveis quando diagnóstico e intervenção terapêutica são iniciados precocemente.

Há duas teorias que tentam explicar o aparecimento do edema encefálico: uma envolve a autorregulação cerebral prejudicada, responsável pelo aumento do fluxo sanguíneo cerebral (FSC) acarretando um edema vasogênico, enquanto a outra envolve um estado inflamatório sistêmico causando uma disfunção endotelial com hipoperfusão cerebral levando a um edema citotóxico. Porém ainda não há consenso na literatura (SCHWARTZ RB, et al., 2000; FITTRO K e DIZON R, 2018).

Os dados epidemiológicos e número de estudos globais da PRES são incertos. Alguns relatos de casos descreveram essa síndrome em uma ampla faixa etária desde os 2 anos a até 90 anos, com a maioria dos casos ocorrendo em jovens e adultos de meia-idade. Observou-se uma marcada preponderância feminina, o que pode refletir alguma das causas subjacentes, porém dados correlacionando com complicações gestacionais ou puerperais são escassos (RIJAL JP, et al., 2014; TRIKHA A, et al., 2014; ARACKI-TRENKIC A, et al., 2016; CAMARGO EC, et al., 2019).

As condições associadas ao desenvolvimento da PRES são medicações, principalmente quimioterápicos e imunobiológicos (11-61\%), doenças autoimunes (8-10\%), hipertensão (6-72\%), infecção/sepse (8-24\%), pré-eclâmpsia e eclâmpsia (7-20\%). A principal anormalidade encontrada em todos os casos é o edema vasogênico cerebral secundário a alterações da perfusão sanguínea cerebral e disfunção da barreira hematoencefálica (LEGRIEL S, et al., 2012; ALVES DR, et al., 2016; SHANKAR J e BANFIEL J. 2017).

A apresentação clínica típica da PRES pode ser de início aguda ou subaguda, associada a sinais e sintomas como hipertensão, cefaleia, (geralmente bilateral), distúrbios visuais (visão borrada, negligência visual, hemianopsia homônima, alucinações visuais e cegueira cortical), convulsões (muitas vezes múltiplas, com estado epiléptico possível; período pós-ictal prolongado e mudanças comportamentais, como confusão e delirium), náusea, vômitos, alteração do nível de consciência, alteração sensorial, sinais neurológicos focais, hiperreflexia simétrica, diminuição da força muscular e coordenação prejudicada dos membros (LEGRIEL S, et al., 2012; ALVES DR, et al., 2016; HU D, et al., 2018). 
São considerados quadros mais graves quando associados a insuficiência neurológica definida por qualquer distúrbio neurológico de origem central como comprometimento da consciência, convulsão com ou sem estado epiléptico, sinal focal, encefalopatia e sinais meníngeos, que exigem tratamento intensivo para monitorização e suporte de vida (ALVES DR, et al., 2016).

A PRES também é considerada uma emergência obstétrica, frequentemente ocorrendo em gestantes ou puérperas geralmente nas primeiras 48 horas pós-parto, usualmente secundária a algum dos distúrbios hipertensivos específicos da gestação como hipertensão, pré-eclâmpsia, eclâmpsia e síndrome marcada por Hemólise - Elevação das Enzimas Hepáticas e Plaquetopenia (HELLP) (MATTHYS LA, et al., 2004; SIBAI BM, 2005).

Na literatura, $7-20 \%$ das pacientes com PRES foram relatadas como tendo pré-eclâmpsia ou eclâmpsia. Não é possível afirmar se estabelecem uma relação de causa e efeito ou sobreposição de fatores de risco, pois a PRES nessas pacientes costumam cursar com cefaleia, crises convulsivas, alterações visuais e alteração do nível de consciência, porém não há uma manifestação clínica específica relacionada a gestantes e puérperas (TRIKHA A, et al., 2014).

O diagnóstico é realizado na presença das condições clínicas acima citadas, associadas aos achados característicos nos exames de imagem, principalmente na ressonância magnética (RNM). A apresentação imaginológica típica da PRES envolve edema vasogênico generalizado e geralmente reversível, afetando predominantemente a substância branca subcortical dos lobos occipital e parietal do cérebro, mais comumente simétrico e bilateral, podendo apresentar-se assimétrico ou unilateral; podendo ainda ocorrer em lobos frontais, temporal, núcleos da base, diferente do que se pensava inicialmente.

São lesões isointensas ou hipointensas nos cortes em T1 (ficam pretas ou cinzas) e hiperintensas em T2 (ficam brancas), sendo mais específicos e sensíveis que os encontrados na tomografia computadorizada (TC), com áreas de hipodensidade difusa da substância branca. A anatomia de partes moles e gordura são melhor visualizadas em T1, pois apresentam-se "brilhantes", (hiperintensas), enquanto em T2 é melhor visualizado líquidos e lesões como tumores, inflamação, edema, traumas, pois nessa ponderação essas imagens que se apresentam hiperintensas (SCHWARTZ RB, et al., 2000).

O sucesso do manejo terapêutico e prevenção de complicações reside principalmente no reconhecimento desses achados clínico-radiológicos da PRES. A terapêutica, pauta-se no diagnóstico precoce e tratamento da causa base, com estabilização clínica, prevenção e tratamento de convulsões, controle pressórico, intervenções para manter o bem-estar materno fetal (remover - ou pelo menos reduzir - os fatores desencadeantes do quadro da PRES), identificar e intervir em possíveis complicações para o binômio mãefeto (MATTHYS LA, et al., 2004; ALVES DR, et al., 2016).

Mais importante, é a intervenção no curso natural da doença, com medidas de controle dos fatores de risco que levarão ao desenvolvimento da PRES, controle da pressão arterial sistêmica com bloqueadores do canal de cálcio, vasodilatadores direto como hidralazina ou nifedipino; controle da pressão intracraniana com manitol e/ou neuro-proteção com sulfato de magnésio (MATTHYS LA, et al., 2004; SIBAI BM 2005). Com diagnóstico e tratamento realizados em tempo hábil, a maioria das pacientes se recupera em duas semanas, apresentando reversibilidade dos sintomas em cinco a oito dias e as alterações radiológicas demoram um pouco mais. Entretanto o atraso no diagnóstico e ou manejo apropriados podem levar a um dano cerebral permanente.

O resultado fatal raramente ocorre e pode ser causado pelo aumento da pressão intracraniana, geralmente em decorrência do edema cerebral progressivo, hemorragia intracerebral ou condições e complicações da causa subjacentes. Quando adequadamente tratadas, as recidivas acontecem em até 3,8\% geralmente provocadas pelo controle inadequado da pressão arterial ou de outra causa base, destacando um acompanhamento e gerenciamento eficaz e duradouro dos fatores de risco (ALVES DR, et al., 2016).

Uma vez que essa síndrome é subdiagnosticada no contexto do ciclo gravídico puerperal, objetiva-se com esta revisão, elencar as principais características fisiopatológicas, clínicas, diagnósticas e manejo terapêutico da Síndrome da Encefalopatia Posterior Reversível em gestantes e puérperas para que possa se identificar e intervir mais precocemente nos casos de PRES, evitando assim complicações irreversíveis e desfechos fatais. 


\section{MÉTODOS}

Esse trabalho foi elaborado a partir de uma revisão da literatura na base de dados CAPES no dia 02/03/2020. As palavras-chave utilizadas foram "PRES", "Posterior Reversible Encephalopathy Syndrome", "Posterior Leukoencephalopathy Syndrome" e "Pregnancy", encontrados 607 artigos, com o critério de inclusão, "Pregnancy", resultando em 90 artigos.

Foram utilizados como critérios de inclusão, artigos que descreviam em seus resumos quadro clínico, fatores de risco, diagnóstico, manejo terapêutico e prognóstico da PRES no ciclo gravídico puerperal. Foram utilizados como critérios de exclusão: artigos que não possuíam resumos; artigos duplicados na base de dados da pesquisa; artigos que não se referiam a PRES no ciclo gravídico puerperal em humanos; artigos que se referiam apenas a hipertensão, tratamento e seu manejo durante a gestação; artigos que se referiam apenas a fisiopatologia da pré-eclâmpsia e eclâmpsia; artigos que se referiam apenas a doenças cerebrovasculares na gestação; artigos que se referiam apenas a cefaleia secundária na gestação; artigo na íntegra escrito em francês; artigo na íntegra escrito em bósnio; sem acesso ao artigo na íntegra pela plataforma CAPES.

Ao final, essa revisão se pautou em 50 artigos sobre a PRES no ciclo gravídico puerperal no intervalo de 2003-2019, com 29 relatos de caso, 11 estudos retrospectivos, 7 revisões de literatura e 3 estudos prospectivos (Figura 1).

Figura 1 - Fluxograma do processo de seleção dos artigos para a revisão.

\section{Pesquisa na plataforma CAPES pelos termos: "PRES" + "Posterior Reversible Encephalopathy Syndrome" + "Posterior Leukoencephalopathy Syndrome" + "Pregnancy" = 607}

$\longrightarrow$\begin{tabular}{l|l|}
\hline Estudos relacionados com o tópico: \\
Relato de caso: 29 \\
Estudo retrospectivo: 11 \\
Revisão de literatura: $\mathbf{0 7}$ \\
Estudo prospectivo: $\mathbf{0 3}$ \\
Total $\mathbf{5} \mathbf{5 0}$ \\
\hline
\end{tabular}

\section{Incluído "Pregnancy" = 90}

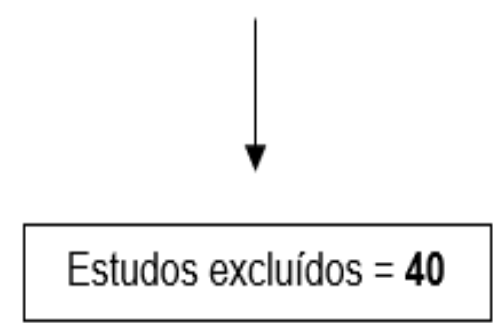

Fonte: Green MCTP, et al., 2020.

\section{RESULTADOS E DISCUSÃO}

No Quadro 1, apresenta a distribuição dos 50 artigos selecionados quanto ao ano, autor, título e tipo de estudo. As publicações estão dispostas em ordem cronológica decrescente. 


\section{Revista Eletrônica Acervo Saúde / Electronic Journal Collection Health ISSN 2178-2091}

Quadro 1 - Artigos selecionados para a revisão bibliográfica, segundo ano de publicação, autores, tipo de estudo e resultado do estudo

\begin{tabular}{|c|c|c|c|c|}
\hline $\mathbf{N}^{\circ}$ & Ano & Autores & Tipo de estudo & Resultados do estudo \\
\hline 01 & 2019 & Camargo EC, et al. (2019); & Revisão de literatura & $\begin{array}{l}\text { Concluíram que as complicações da PRES em pacientes não-obstétricos podem ser mais } \\
\text { graves do que os pacientes com PRES no período gestacional, devido a severidade das } \\
\text { comorbidades subjacentes. }\end{array}$ \\
\hline 02 & 2019 & Dong X e Nao J. (2019); & $\begin{array}{l}\text { Retrospectivo } \\
\qquad \mathrm{N}=56\end{array}$ & $\begin{array}{l}\text { Concluíram que cefaleia e convulsões frequentes estão independentemente associadas a } \\
\text { apresentação atípica da PRES. E, quando presente, a restrição de difusão na RM, pode auxiliar } \\
\text { na predição de pior prognóstico. }\end{array}$ \\
\hline 03 & 2019 & $\begin{array}{l}\text { Katsevman GA, et al. } \\
\text { (2019); }\end{array}$ & Relato de caso & $\begin{array}{l}\text { Descreve uma abordagem mais agressiva para descompressão através de craniectomia. E após } \\
\text { dois anos paciente ainda apresenta cefaleias ocasionais, sem outras complicações. }\end{array}$ \\
\hline 04 & 2018 & Garg R, et al. (2018); & Revisão de Literatura & $\begin{array}{l}\text { Descreve a PRES como uma complicação grave da eclâmpsia, com o reconhecimento e } \\
\text { tratamento precoce ajudam a prevenir algumas de suas sequelas devastadoras A maioria dos } \\
\text { pacientes apresentou melhora clínica e resolução espontânea. }\end{array}$ \\
\hline 05 & 2018 & Hirashima H, et al. (2018); & Relato de caso & $\begin{array}{l}\text { É necessária melhor elucidação do papel do anticorpo fosfolipídeo lgM nas doenças } \\
\text { cerebrovasculares durante a gravidez e período puerperal. Ainda assim houve resolução } \\
\text { completa das alterações apresentadas. }\end{array}$ \\
\hline 06 & 2018 & Hu D, et al. (2018); & Relato de caso & $\begin{array}{l}\text { Descreve que a hemorragia intracraniana em pequena quantidade pode ter evolução favorável, } \\
\text { mesmo com estudos que demonstram aumento da morbimortalidade materna. Houve resolução } \\
\text { completa dos sintomas, sem terapia anti-hipertensiva e sem terapia antiepiléptica }\end{array}$ \\
\hline 07 & 2018 & Mansingh S, et al. (2018); & Relato de caso & $\begin{array}{l}\text { Descreve a importância do diagnóstico diferencial com eclâmpsia e PRES pós-natal, no qual a } \\
\text { história clínica sem hipertensão e o exame de imagem auxiliaram nos diagnósticos. As pacientes } \\
\text { ficaram assintomáticas, sem sequelas neurológicas }\end{array}$ \\
\hline 08 & 2018 & Rajiv KR, et al. (2018); & Prospectivo $\mathrm{N}=10$ & $\begin{array}{l}\text { Em } 3 \text { pacientes ( } 30 \%) \text { a causa do estado epiléptico foi a PRES. Concluindo-se que o manejo do } \\
\text { estado epiléptico depende da etiologia, idade gestacional e comorbidades maternas. Com o } \\
\text { manejo adequado dessas pacientes, } 80 \% \text { delas apresentaram recuperação para o estado basal. }\end{array}$ \\
\hline 09 & 2018 & Singh S, et al. (2018); & Relato de caso & $\begin{array}{l}\text { É necessário ter conhecimento sobre a PRES e alto nível de suspeição para reconhecê-la. O } \\
\text { diagnóstico oportuno ajuda a prevenir as sequelas a longo prazo dessa condição reversível. } \\
\text { Resolução total dos sintomas, sem sequelas }\end{array}$ \\
\hline
\end{tabular}

REAS / EJCH | Vol.12(11) | e4724 | DOI: https://doi.org/10.25248/reas.e4724.2020 Página 5 de 15 


\begin{tabular}{|c|c|c|c|c|}
\hline $\mathbf{N}^{\circ}$ & Ano & Autores & Tipo de estudo & Resultados do estudo \\
\hline 10 & 2017 & $\begin{array}{l}\text { Mescher C e Slungaard A. } \\
(2017) \text {; }\end{array}$ & Relato de caso & $\begin{array}{l}\text { Paciente puérpera desenvolveu PRES exposta ao tratamento de Leucemia linfoide aguda com } \\
\text { ciclofosfamida e metotrexato. Após ter sido novamente exposta a essas drogas não apresentou } \\
\text { complicações neurológicas subsequentes. } \\
\text { Houve remissão dos sintomas neurológicos e achados nos exames de imagem, porém veio a } \\
\text { óbito } 11 \text { meses após o diagnóstico do câncer devido a um embolo pulmonar }\end{array}$ \\
\hline 11 & 2017 & Takahashi H, et al. (2017); & Relato de caso & $\begin{array}{l}\text { Frisa a importância de Importância de se investigar outras queixas visuais como única } \\
\text { manifestação clínica de PRES, neste caso a paciente apresentou acromatopsia. Após } 4 \\
\text { semanas houve reversão de todo o quadro neurológico sem sequelas }\end{array}$ \\
\hline 12 & 2017 & $\begin{array}{l}\text { Tetsuka S e Nonaka H } \\
\text { (2017); }\end{array}$ & Relato de Caso & $\begin{array}{l}\text { Paciente desenvolveu síndrome HELLP concomitante a PRES. Foi instituída terapêutica } \\
\text { adequada, com bom prognóstico. Após } 21 \text { dias paciente apresentou reversibilidade de todas as } \\
\text { alterações encontradas, sem complicações. }\end{array}$ \\
\hline 13 & 2017 & Wen Y, et al. (2017); & Retrospectivo $\mathrm{N}=36$ & $\begin{array}{l}\text { Descreve a importância do diagnóstico imagem oportuno na PRES e seus diagnósticos } \\
\text { diferenciais que devem ser considerados. O diagnóstico precoce acarreta um manejo mais } \\
\text { apropriado, prevenindo possíveis déficits neurológicos a longo-prazo.30 pacientes } \\
\text { apresentaram reversibilidade dos sintomas, } 4 \text { pacientes apresentaram déficits residuais e } 2 \\
\text { morreram devido a distúrbios de coagulação }\end{array}$ \\
\hline 14 & 2016 & $\begin{array}{l}\text { Aracki-Trenkić A, et al. } \\
(2016) ;\end{array}$ & Revisão de literatura & $\begin{array}{l}\text { Neste estudo preconizam a descontinuação do uso de nitroglicerina intravenosa no tratamento } \\
\text { de hipertensão aguda e encefalopatia hipertensiva }\end{array}$ \\
\hline 15 & 2016 & $\begin{array}{l}\text { Hentschke MR, et al. } \\
(2016) ;\end{array}$ & Relato de caso & $\begin{array}{l}\text { Descrevem que a PRES pode apresentar evolução satisfatória se diagnosticada e tratada } \\
\text { adequadamente. Houve remissão completa dos sintomas e reversão do quadro clínico inicial. }\end{array}$ \\
\hline 16 & 2016 & More A, et al. (2016); & Relato de caso & $\begin{array}{l}\text { Este estudo refere como causa da perda aguda da visão no período pós-parto em pacientes } \\
\text { HIV positivas a PRES e a meningite criptocócica. }\end{array}$ \\
\hline 17 & 2015 & Cozzolino M, et al. (2015); & Relato de caso & $\begin{array}{l}\text { Neste estudo utilizam a RM, padrão-ouro para o diagnóstico de PRES e como pode ser útil no } \\
\text { diagnóstico diferencial. O objetivo do manejo terapêutico é o controle pressórico, prevenir e } \\
\text { tratar convulsões. Dessa forma há melhora progressiva do estado de consciência com resolução } \\
\text { de déficit neurológico, normalização da pressão arterial e reversibilidade das alterações no } \\
\text { exame de imagem }\end{array}$ \\
\hline
\end{tabular}

REAS / EJCH | Vol.12(11) | e4724 | DOI: https://doi.org/10.25248/reas.e4724.2020 Página 6 de 15 


\section{Revista Eletrônica Acervo Saúde / Electronic Journal Collection Health ISSN 2178-2091}

\begin{tabular}{|c|c|c|c|c|}
\hline $\mathbf{N}^{\circ}$ & Ano & Autores & Tipo de estudo & Resultados do estudo \\
\hline 18 & 2015 & $\begin{array}{l}\text { Hammer ES e Cipolla MJ } \\
(2015) \text {; }\end{array}$ & Revisão de literaura & $\begin{array}{l}\text { Evidenciou melhor prognóstico e quadro clínico inicial menos severo em pacientes com pré- } \\
\text { eclâmpsia e PRES quando comparadas a PRES de outras etiologias associados a melhor } \\
\text { resolução do quadro sem complicações e melhor prognóstico a longo prazo. }\end{array}$ \\
\hline 19 & 2015 & Kurdoglu Z, et al. (2015); & Retrospectivo $\mathrm{N}=81$ & $\begin{array}{l}\text { Neste estudo } 45 \text { pacientes apresentaram PRES. } 27 \text { (60\%) apresentaram cefaleia associada a } \\
\text { alterações visuais; Proteinúria, maior IMC, maior tempo de internação foram identificados } \\
\text { nessas pacientes associados a resultados neonatais adversos são mais comuns nestas } \\
\text { pacientes. }\end{array}$ \\
\hline 20 & 2015 & Wang Y, et al. (2015); & Relato de caso & $\begin{array}{l}\text { Esclarece a importância das características e fatores variáveis de apresentação da PRES e sua } \\
\text { associação com cegueira bilateral. }\end{array}$ \\
\hline 21 & 2014 & Akins PT, et al. (2014); & Prospectivo $\mathrm{N}=05$ & $\begin{array}{l}\text { Demostrou que intervenções precoces e agressivas, incluindo atendimento neurológico } \\
\text { adequado e tratamento cirúrgico refletem em um prognóstico melhor que o esperado. Todas as } \\
\text { pacientes evoluíram bem, apenas uma continuou apresentando déficit de campo visual. }\end{array}$ \\
\hline 22 & 2014 & Alhilali LM, et al. (2014); & Retrospectivo $\mathrm{N}=47$ & $\begin{array}{l}\text { Toxemia da gestação (TOP) foi identificada em } 14 / 47 \text { dos pacientes (30\%), sendo identificada } \\
\text { como único fator de proteção significativa, com } 75 \% \text { risco diminuído de morte materna }\end{array}$ \\
\hline 23 & 2014 & Dag ZO, et al. (2014); & Relato de caso & $\begin{array}{l}\text { Uma gravidez oculta pode levar ao atraso do diagnóstico e terapêutica, aumento o risco de } \\
\text { complicações materna e fetais. } \\
\text { Houve reversibilidade total dos sintomas, porém o feto morreu } 03 \text { dias após o parto }\end{array}$ \\
\hline 24 & 2014 & $\begin{array}{l}\text { Karuppannasamy D, et al. } \\
(2014) \text {; }\end{array}$ & Retrospectivo $\mathrm{N}=10$ & $\begin{array}{l}\text { Refere a prevalência de alterações visuais em } 5 \text { (50\%) pacientes com PRES associadas a } \\
\text { eclampsia no pós-parto. } \\
100 \% \text { apresentaram remissão dos sintomas sem sequelas }\end{array}$ \\
\hline 25 & 2014 & Oyinloye OI, et al. (2014); & Relato de caso & $\begin{array}{l}\text { Descrevem a importância do reconhecimento dos achados típicos de imagem para diagnosticar } \\
\text { PRES }\end{array}$ \\
\hline 26 & 2014 & Postma IR, et al. (2014); & Revisão de literatura & $\begin{array}{l}\text { Neste estudo elencam a possibilidade de que a PRES na população obstétrica está associada } \\
\text { a uma evolução menos grave e ocorrência menos frequente de sequelas a longo prazo, pois a } \\
\text { maioria apresentou reversibilidade do quadro. }\end{array}$ \\
\hline
\end{tabular}

REAS / EJCH | Vol.12(11) | e4724 | DOI: https://doi.org/10.25248/reas.e4724.2020 Página 7 de 15 


\section{Revista Eletrônica Acervo Saúde / Electronic Journal Collection Health ISSN 2178-2091}

\begin{tabular}{|c|c|c|c|c|}
\hline $\mathbf{N}^{\circ}$ & Ano & Autores & Tipo de estudo & Resultados do estudo \\
\hline 27 & 2014 & Razmara A, et al. (2014); & Revisão de literatura & $\begin{array}{l}\text { A compreensão dos mecanismos subjacentes e tratamento precoce dos sinais e sintomas na } \\
\text { PRES pode ajudar a prevenir eventos vasculares agudos antes do parto e o período pós-parto. } \\
\text { Pois a maioria das pacientes apresenta curso autolimitado com resolução completa de } \\
\text { anormalidades clínicas e de imagem. }\end{array}$ \\
\hline 28 & 2014 & Rijal JP, et al. (2014); & Relato de caso & $\begin{array}{l}\text { Enfatizam a importância do diagnóstico de PRES no período puerperal e terapêutica realizada } \\
\text { para manejo adequado com rápida recuperação do quadro, sem sequelas ou sintomas } \\
\text { residuais. }\end{array}$ \\
\hline 29 & 2014 & Trikha A, et al. (2014); & Revisão de literatura & $\begin{array}{l}\text { A PRES é um desafio diagnóstico devido à apresentação clínica inespecífica e condições } \\
\text { clínicas associadas da paciente, especialmente durante a gravidez. Podendo cursar com } \\
\text { complicações como isquemia, hemorragia e herniação cerebral. }\end{array}$ \\
\hline 30 & 2013 & Brewer J, et al. (2013); & Retrospectivo $\mathrm{N}=47$ & $\begin{array}{l}\text { Demonstram que a terapia direcionada a prevenção ou reversão da patogênese da PRES pode } \\
\text { impedir ou facilitar a recuperação da eclâmpsia. Todas as pacientes do estudo apresentaram } \\
\text { reversão dos sintomas neurológicos entre } 9-135 \text { horas após o inícios dos mesmos. }\end{array}$ \\
\hline 31 & 2013 & Youssoufa M, et al. (2013); & Relato de caso & $\begin{array}{l}\text { Relatam a importância do segmento após a PRES e suas possíveis complicações. Uma paciente } \\
\text { após } 9 \text { meses desenvolveu epilepsia secundária a PRES. }\end{array}$ \\
\hline 32 & 2012 & Demir BC, et al. (2012); & Retrospectivo $\mathrm{N}=62$ & $\begin{array}{l}\text { Foi evidenciado que o manitol não contribui para o prognóstico das pacientes, além do } \\
\text { tratamento ser mais prolongado gerando custos adicionais em comparação com pacientes em } \\
\text { uso de sulfato de magnésio que apresentaram melhor prognóstico e menor tempo de } \\
\text { internação. }\end{array}$ \\
\hline 33 & 2012 & Legriel S, et al. (2012); & Retrospectivo $\mathrm{N}=70$ & $\begin{array}{l}\text { Foi evidenciado que a Toxemia da Gravidez (TOP) está independentemente associada a } \\
\text { prognóstico mais favorável ao término dos } 90 \text { dias em pacientes que apresentem PRES severa, } \\
\text { com índice de mortalidade maternal após eclampsia de } 1 \% \text {. }\end{array}$ \\
\hline 34 & 2012 & Liman TG, et al. (2012); & Retrospectivo $\mathrm{N}=96$ & $\begin{array}{l}\text { Pacientes com pré-eclâmpsia/eclâmpsia apresentaram sintomas iniciais mais leves e melhor } \\
\text { reversibilidade do edema e menor frequência de lesões estruturais graves. Pacientes com PRES } \\
\text { devido a outras causas são afetados com maior gravidade tanto clínica quanto } \\
\text { radiologicamente. Concluindo que a PRES relacionada ao período gestacional apresenta melhor } \\
\text { reversibilidade do edema cerebral e menor frequência de lesões cerebrais estruturais graves } \\
\text { que a PRES por outras causas. }\end{array}$ \\
\hline
\end{tabular}

REAS / EJCH | Vol.12(11) | e4724 | DOI: https://doi.org/10.25248/reas.e4724.2020 Página 8 de 15 


\section{Revista Eletrônica Acervo Saúde / Electronic Journal Collection Health ～ISSN 2178-2091}

\begin{tabular}{|c|c|c|c|c|}
\hline $\mathbf{N}^{\circ}$ & Ano & Autores & Tipo de estudo & Resultados do estudo \\
\hline 35 & 2012 & Orehek EK, et al. (2012); & Relato de caso & $\begin{array}{l}\text { Esse caso ilustra complicações da hipertensão intracraniana no período gravídico puerperal, } \\
\text { desafio diagnóstico e manejo terapêutico. A paciente manteve leve dismetria em membro } \\
\text { superior esquerdo. }\end{array}$ \\
\hline 36 & 2012 & Siddiqui TS, et al. (2012); & Relato de caso & $\begin{array}{l}\text { Importância do reconhecimento dos achados de imagem característicos são fundamentais para } \\
\text { diagnosticar PRES e medidas terapêuticas para a remissão quase completa das anormalidades } \\
\text { apresentadas à admissão. }\end{array}$ \\
\hline 37 & 2011 & Achar SK, et al. (2012); & Relato de caso & $\begin{array}{l}\text { Enfatiza a necessidade de diagnóstico precoce e tratamento imediato da PRES para evitar } \\
\text { sequelas neurológicas de curto e longo praz }\end{array}$ \\
\hline 38 & 2011 & Hegde HV, et al. (2011); & Relato de caso & $\begin{array}{l}\text { Discute-se a opção da via de parto em pacientes com PRES anteparto. Qual via seria mais } \\
\text { segura, uma vez que nesses casos deve-se se considerar na tomada de decisão o bem-estar } \\
\text { materno e fetal. }\end{array}$ \\
\hline 39 & 2011 & Matsubara S, et al. (2011); & Relato de caso & $\begin{array}{l}\text { Esse caso foi considerado "atípico", pois não foi possível identificar uma causa base para a } \\
\text { PRES dessa paciente, apenas a gravidez. } \\
\text { Sinais, sintomas e os achados da ressonância foram espontaneamente reversíveis, sem a } \\
\text { necessidade de intervenção terapêutica invasiva }\end{array}$ \\
\hline 40 & 2011 & Wagner SJ, et al. (2011); & Retrospectivo $\mathrm{N}=13$ & $\begin{array}{l}\text { Discute a importância da implementação de um alvo terapêutico para melhor manejo e controle } \\
\text { da pressão arterial nas gestantes que desenvolvem PRES. }\end{array}$ \\
\hline 41 & 2010 & Aygün BK, et al. (2010); & Relato de caso & $\begin{array}{l}\text { O reconhecimento da PRES precoce, permite que tratamento adequado seja realizado, } \\
\text { diminuindo possíveis complicações }\end{array}$ \\
\hline 42 & 2010 & Barbosa AS, et al. (2010); & Prospectivo $\mathrm{N}=112$ & $\begin{array}{l}26 \text { pacientes (41\%) apresentarem PRES cursando com menor índice resistência de artéria } \\
\text { sendo este um biomarcador relevante da PRES na pré-eclâmpsia grave. }\end{array}$ \\
\hline 43 & 2009 & Gasco J, et al. (2009); & Relato de caso & $\begin{array}{l}\text { Relatam a singularidade e individualização do manejo de eventos hemorrágicos agudos } \\
\text { relacionados a PRES como Derivação Lombo-Peritoneal }\end{array}$ \\
\hline
\end{tabular}

REAS / EJCH | Vol.12(11) | e4724 | DOI: https://doi.org/10.25248/reas.e4724.2020 Página 9 de 15 


\section{Revista Eletrônica Acervo Saúde / Electronic Journal Collection Health ～ISSN 2178-2091}

\begin{tabular}{|c|c|c|c|c|}
\hline $\mathbf{N}^{\circ}$ & Ano & Autores & Tipo de estudo & Resultados do estudo \\
\hline 44 & 2008 & Peng WX, et al. (2008); & Relato de caso & $\begin{array}{l}\text { Caso de síndrome HELLP complicado por PRES pós-parto com achados de neuroimagem } \\
\text { "atípica". O reconhecimento e início do manejo desta síndrome podem diminuir riscos de lesões } \\
\text { irreversíveis. } \\
\text { Paciente apresentou resolução dos sinais e sintomas, sem sequelas }\end{array}$ \\
\hline 45 & 2008 & $\begin{array}{l}\text { Pratap JN e Down JF } \\
\text { (2008); }\end{array}$ & Relato de caso & $\begin{array}{l}\text { Paciente apresentou quadro clínico de PRES de maior gravidade, cursando com rebaixamento } \\
\text { do nível de consciência. Foi manejada em tempo hábil. Apresentou atividade epileptiforme focal } \\
\text { no lobo temporal esquerdo, com crises parciais simples associado a déficit de memória em } \\
\text { remissão. }\end{array}$ \\
\hline 46 & 2007 & Long TR, et al. (2007); & Relato de caso & $\begin{array}{l}\text { Concluem a importância do pronto reconhecimento e tratamento da PRES podendo evitar } \\
\text { lesões irreversíveis para a paciente. } \\
\text { Houve remissão clínica e das alterações dos exames de imagem após } 7 \text { meses. }\end{array}$ \\
\hline 47 & 2007 & Onderoglu LS, et al. (2007); & Relato de caso & $\begin{array}{l}\text { Concluíram que o envolvimento cortical cerebral pode ser um componente importante da pré- } \\
\text { eclâmpsia/eclâmpsia e distúrbios visuais podem ser um sinal de alerta de PRES. Todos os } \\
\text { achados voltaram completamente ao normal. }\end{array}$ \\
\hline 48 & 2007 & $\begin{array}{l}\text { Powell ES e Goldman MJ } \\
\text { (2007); }\end{array}$ & Relato de caso & $\begin{array}{l}\text { Concluíram que o tratamento adequado no cenário agudo permite a restauração completa da } \\
\text { função cognitiva a longo prazo, porém paciente manteve-se hipertensa. }\end{array}$ \\
\hline 49 & 2005 & Finocchi V, et al. (2005); & Relato de caso & $\begin{array}{l}\text { Sugerem que as sequências FLAIR e DWI deveriam ser incluídas nos protocolos de RM para } \\
\text { suspeita de PRES, porque ajudam não apenas para a identificação correta das lesões, mas } \\
\text { também para o manejo e acompanhamento adequados de lesões isquêmicas irreversíveis das } \\
\text { pacientes. }\end{array}$ \\
\hline 50 & 2003 & Servillo G, et al. (2003); & Retrospectivo $\mathrm{N}=04$ & $\begin{array}{l}\text { Neste estudo, } 4 \text { pacientes desenvolveram PRES com quadro clínico típico. Foi instituída } \\
\text { terapêutica, } 3 \text { pacientes apresentaram reversibilidade do quadro e dos achados de imagem, } \\
\text { porém } 1 \text { paciente desenvolveu hemorragia subaracnóidea indo a óbito. }\end{array}$ \\
\hline
\end{tabular}

Fonte: Green MCTP, et al., 2020.

REAS / EJCH | Vol.12(11) | e4724 | DOI: https://doi.org/10.25248/reas.e4724.2020 Página 10 de 15 
A Síndrome da Encefalopatia Posterior Reversível é uma síndrome de etiopatogenia multifatorial, com fatores de riscos relacionados ao período gravídico e puerperal próprios, que cursam com particularidades e singularidades em suas manifestações clínicas, diagnósticas e abordagens terapêuticas, o que não era claro e nítido até abordarem em suas publicações (COZZOLINO M, et al., 2015; WEN Y, et al., 2017).

Quanto aos aspectos fisiopatológicos do desenvolvimento dessa síndrome, mesmo nessa revisão da literatura, ainda não existe um consenso definitivo e único dentre os pesquisadores; contudo, duas hipóteses são atualmente mais aceitas e utilizadas para explicar os meios de desenvolvimento e acometimento neurológico clínico e radiológico, não significando que os dois processos não se correlacionem.

Onderoglu LS, et al. (2007) e Achar SK, et al. (2011) consideram como mecanismo fisiopatológico a ocorrência de um edema vasogênico na substância branca do SNC, decorrente da quebra da barreira hematoencefálica por perda dos mecanismos autorregulatórios, principalmente por variações agudas da pressão arterial, compartilhando o mesmo mecanismo fisiopatológico da eclâmpsia.

No entanto, essa teoria falha em explicar por que a PRES pode ocorrer em paciente com pressão arterial levemente elevada ou mesmo normotenso, uma vez que a síndrome, sua extensão, e a gravidade do edema não estão diretamente correlacionados aos valores pressóricos. A segunda fisiopatologia mais aceita evidencia a origem do edema, por disfunção do endotélio vascular cerebral, através de espasmos arteriolares, com consequente aumento abrupto da pressão arterial e desencadeamento de isquemia do tecido encefálico e edema citotóxico (COZZOLINO M, et al., 2015; WEN Y, et al., 2017).

Liman et al. (2012) revelam, apesar desse estado inflamatório sistêmico, nas pacientes com PRES por fatores como pré-eclâmpsia e eclâmpsia apresentam marcadores de inflamação como Proteína C Reativa (PCR) em valores menores quando comparadas a PRES desencadeada por outros fatores.

A partir dessas duas teorias fisiopatológicas, evidencia-se que elas têm em comum fatores de risco para o seu desenvolvimento, os quais são o diagnóstico das doenças hipertensivas exclusivas da gestação e suas complicações associadas como a pré-eclâmpsia e eclâmpsia, podendo em até $12 \%$ dos casos de préeclâmpsia evoluíram para PRES, e eclâmpsia com incidência ainda maior (BREWER J, et al., 2013; HAMMER ES, et al., 2015; ARACKI-TRENKIC A, et al., 2016).

Wen Y, et al. (2017), Kurdoglu Z, et al. (2015), Liman TG, et al. (2012), Brewer J, et al. (2013) e Achar SK, et al. (2011) apontam que, nas pacientes com PRES relacionada à pré-eclâmpsia e eclâmpsia os sintomas mais frequentes relatados foram a cefaleia, $81 \%$ de ocorrência, seguida imediatamente por crises convulsivas, $73 \%$, a alteração do nível de consciência em $57 \%$, náuseas e vômitos $47 \%$ e, por fim, $33 \%$ de alterações visuais estiveram presentes em $33 \%$.

Há relato de concomitância entre a ocorrência de cefaleia com alterações visuais em $60 \%$ das pacientes que apresentam PRES decorrente de eclâmpsia, o que traz um importante fato: maior ocorrência dos fenômenos visuais associados à PRES no período gravídico-puerperal (KURDOGLU Z, et al., 2015). Cozzolino M, et al. (2015), sintetizam que independente da presença de cefaleia ou demais sinais e sintomas que ocorrem na PRES relacionado à gestação ou puerpério, raramente a PRES se desenvolverá na ausência de crises convulsivas.

Os achados de alterações visuais isolados nas pacientes gestantes ou puérperas são considerados atualmente e em consenso os mais raros de se manifestarem, e na grande maioria dos casos são transitórios, tendo regressão em horas a semanas. As alterações visuais reportadas variam entre déficit da acuidade visual, fotofobia, fotopsia, acromatopsia e cegueira cortical, caracterizada pelos reflexos pupilar e exame de fundoscopia fisiológicos (ARACKI-TRENKIC A, et al., 2016; RAJIV KR, et al., 2018; WEN Y, et al., 2017). Karuppannasamy M, et al. (2014) identificaram a presença de alterações visuais, cegueira cortical súbita, em $50 \%$ das pacientes estudadas.

Em relação a investigação diagnóstica, a PRES possui essencialmente um caráter clínico-radiológico, sendo seus principais achados em neuroimagem o edema vasogênico generalizado nas regiões posteriores da substância branca subcortical dos lobos parietais e occipitais do encéfalo, e mais atualmente tem-se considerado edema no córtex frontal, temporal e até mesmo porções do tronco cerebral. 
Mais comumente esses achados são simétricos e bilaterais, podendo apresentar-se assimétrico ou unilateral, em sua maioria são transitórios, regredindo entre horas até semanas após instituição terapêutica (BREWER J, et al., 2013; ARACKI-TRENKIC A, et al., 2016).

Pode-se, inicialmente, utilizar a tomografia computadorizada (TC), em especial à admissão da paciente, para excluir outras causas, porém o padrão-ouro para se diferenciar as lesões da PRES é a ressonância magnética (RNM) nas versões T1, T2, Imagem Ponderada por Difusão (DWI) e Recuperação por Inversão de Atenuação de Fluido (FLAIR).

Uma que permite melhores resultados como a visualização precoce das lesões características de PRES, que podem ser indetectáveis em até $50 \%$ dos exames de TC, além de permitirem a avaliação da regressão ou não das lesões primariamente formadas no evento inicial da PRES, semanas após sua ocorrência, além de diferenciar o diagnóstico com outras possíveis causas de edema cerebral ou entidades clínicas diferenciais como hemorragia intracraniana, encefalopatia isquêmica, trombose dos seios venosos cerebrais, dissecção de artéria cerebral, doenças e infecções do sistema nervoso central (PENG WX, et al., 2008; LIMAN TG, et al., 2012; ALHILALI LM, et al., 2014).

Finocchi V, et al. (2005) em seu estudo já sugeriam que as sequências FLAIR e DWI deveriam ser incluídas nos protocolos de RNM para suspeita de PRES, porque ajudam não apenas na identificação correta das lesões, mas também no manejo e acompanhamento adequados de possíveis lesões isquêmicas irreversíveis.

Dong X e Nao J (2019) em seu estudo retrospectivo identificou que a restrição de difusão na RM poderia indicar um pior prognóstico. Barbosa AS, et al. (2010) demonstrou através do exame doppler das artérias oftálmicas que, com valores progressivamente mais baixos do índice de resistência (IR) da artéria oftálmica, há uma probabilidade progressivamente maior da PRES ocorrer. Sendo, portanto, o IR da artéria oftálmica um biomarcador relevante da PRES na pré-eclâmpsia grave. Esses dados são úteis para a identificação de pacientes com PRES de maior severidade, que necessitem de cuidados mais agressivos e com melhores desfechos materno-fetal.

Assim a estratégia do manejo das pacientes com síndrome da encefalopatia posterior reversível independentemente da etiologia, mas especificamente nessa revisão da literatura, é o tratamento de suporte generalizado, juntamente com a remoção da causa subjacente.

Em pacientes no período gravídico e puerperal, que cursam com pré-eclâmpsia, eclâmpsia ou outras complicações, o manejo ideal é feito através da regulação da pressão arterial com uso de labetalol, hidralazina, nitroprussiato de sódio, diuréticos; controle ou prevenção das convulsões e desenvolvimento de eclâmpsia com o sulfato de magnésio; diminuição de edema cerebral com emprego de manitol e remoção da causa base, no caso, se as pacientes estiverem gestantes, indica-se a resolução obstétrica da gestação.

No caso de síndrome HELLP em dois estudos foram utilizados corticosteroides, a exemplo a dexametasona, mesilato de gabexato e anti-trombina III, com resultados favoráveis (COZZOLINO M, et al., 2015; RAJIV KR, 2018; SINGH S, 2018).

É indicada a descontinuação do uso de nitroglicerina intravenosa no tratamento de hipertensão aguda e encefalopatia hipertensiva devido à piora das manifestações clínicas neurológicas. Evidenciou-se que o uso do manitol não é superior ao uso de sulfato de magnésio para alcançar melhora neurológica, uma vez que o manitol não contribui para o prognóstico das pacientes, além do tratamento e tempo de internação ser mais prolongado. Porém, há normalização mais rápida da função neurológica entre as pacientes com eclâmpsia pré-parto que receberam corticoide para a síndrome HELLP ou maturação pulmonar que as pacientes com eclâmpsia pós-parto (DEMIR BC, et al., 2012; COZZOLINO M, et al., 2015; ARACKI-TRENKIC A, et al., 2016; WEN Y, et al., 2017).

Em pacientes com eclâmpsia e PRES foram identificados fatores em comum como maior pressão arterial média à admissão, maior pressão arterial após o 1 trimestre de gestação, aumento de lactato desidrogenase (DHL), proteinúria, maior índice de massa corporal (IMC), maior tempo de internação e resultados neonatais adversos são mais comuns quando comparadas a gestantes com eclâmpsia sem PRES (BARBOSA AS, et al., 2010; KURDOGLU Z, et al., 2015). 
Por outro lado, a toxemia da gravidez (TOP - "toxemia of pregnancy"), definida em pacientes grávidas ou puérperas com pressão arterial sistólica maior ou igual a $160 \mathrm{mmHg}$ ou diastólica maior ou igual a $110 \mathrm{mmHg}$ em duas ocasiões com uma diferença de 6 horas, enquanto em repouso, proteinúria maior ou igual a $5 \mathrm{~g}$ em uma coleta de urina de 24 horas, ou maior igual a 3+ em duas amostras aleatórias de urina coletadas com 4 h de intervalo ou oligúria menor que $500 \mathrm{~mL}$ em $24 \mathrm{~h}$, distúrbios cerebrais ou visuais, edema/cianose pulmonar, dor epigástrica/quadrante superior direito, função hepática comprometida, trombocitopenia ou restrição do crescimento fetal, foi relacionada a um melhor prognóstico materno-fetal (LEGRIEL S, et al., 2012; ALHILALI LM, et al., 2014).

Assim, pode-se avaliar que a PRES em pacientes que evoluíram em algum momento do período gravídicopuerperal, com pré-eclâmpsia e eclâmpsia apresentam quadro clínico inicial menos severo, de melhor prognóstico, com maior reversibilidade do edema, menor frequência de lesões estruturais graves e menor frequência de lesões a longo prazo quando comparadas a PRES de outras etiologias. Isso se deve provavelmente a severidade das comorbidades subjacentes apresentadas pelos pacientes com PRES nãoobstétrica (HAMMER ES e CIPOLLA MJ, 2015; CAMARGO EC, et al., 2019).

As complicações mais graves a curto prazo encontradas na PRES são: isquemia encefálica, hemorragia intracraniana (HIC) e herniação cerebral. A isquemia sendo o primeiro sinal de irreversibilidade na PRES, a herniação decorrente do edema cerebral, já a hemorragia é uma complicação mais incomum, porém podendo ocorrer na região subaracnóidea ou intraventricular (TRIKHA A, et al., 2014).

Como o próprio nome da PRES diz, quando realizado diagnóstico e manejo adequados e precoces, tende a ter uma evolução reversível e favorável tanto do quadro clínico quanto das alterações nos exames de imagem, o que é corroborado pela maior parte da literatura, porém em alguns estudos revisados, foram notadas algumas sequelas como déficits neurológicos persistentes, óbito materno e fetal (SERVILLO G, et al. 2003; AYGUN BK, et al., 2010; OREHEK EK, et al., 2012; AKINS PT, et al., 2014; KARUPPANNASAMY D, et al., 2014; RAZMARA A, et al., 2014; WEN Y, et al., 2017; RAJIV KR, et al., 2018; KATSEVMAM GA, et al., 2019).

Os resultados de uma intervenção precoce e adequada são: menor morbimortalidade materna e fetal, menores custos aos serviços de saúde e maior qualidade de vida a paciente e sua família (ARACKI-TRENKIC A, et al., 2016).

Por isso quando pensar em PRES em uma paciente gestante ou puérpera? Sugerimos aqui um recurso mnemônico com algumas características que podem ajudar a lembrar da síndrome da encefalopatia posterior reversível no diagnóstico diferencial.

Abordamos fatores desencadeantes, manifestação clínica, achado de imagem e um fator que pode indicar pior prognóstico na ressonância magnética, como recurso prático na urgência e contribuição para um diagnóstico e manejo precoces (Figura 2).

Figura 2 - Mnemônico para PRES.

$\boldsymbol{P} \square$ Pré-eclâmpsia e eclâmpsia
$\boldsymbol{R} \square$ Restrição de difusão na RNM
$\boldsymbol{E} \square$ Edema cerebral
$\boldsymbol{S} \square$ Sintomas visuais.

Fonte: Green MCTP, et al., 2020. 


\section{CONCLUSÃO}

A PRES é uma síndrome de início agudo, sendo importante o reconhecimento das suas características clínicas e diagnósticas, pois, embora essa condição seja geralmente transitória e completamente reversível quando realizado diagnóstico e manejo em tempo hábil, podem ocorrer lesões irreversíveis. Principalmente em um cenário agudo no qual uma paciente puérpera ou gestante se apresente com quadro de cefaleia intensa, alterações visuais ou alteração do nível de consciência, é necessária suspeição diagnóstica da PRES. Mesmo que não haja ainda um consenso em relação ao manejo adequado destas pacientes, visa-se o controle das causas bases que cursam com está síndrome para melhor desfecho materno e fetal. Esperamos que com essa síndrome sendo mais diagnosticada no ciclo gravídico-puerperal, possa-se somar mais dados a literatura e com isso aventar um melhor manejo para estas pacientes, por isso criamos o mnemônico para auxiliar em um cenário de emergência.

\section{REFERÊNCIAS}

1. ACHAR SK, et al. Posterior reversible encephalopathy syndrome at term pregnancy. Indian Journal of Anaesthesia. 2011;55(4):399.

2. AKINS PT, et al. Management and outcomes of malignant posterior reversible encephalopathy syndrome. Clinical Neurology and Neurosurgery. 2014;125:52-7.

3. ALHILALI LM, et al. A multi-disciplinary model of risk factors for fatal outcome in posterior reversible encephalopathy syndrome. Journal of the Neurological Sciences. 2014;347(1-2):59-65.

4. ALVES DR, et al. Posterior Reversible Encephalopathy Syndrome in a pregnant patient: a PRESsing matter. Acta Anaesthesiologica Scandinavica. 2016;60(10):1473-6.

5. ARACKI-TRENKIC A, et al. Atypical presentation of posterior reversible encephalopathy syndrome: Clinical and radiological characteristics in eclamptic patients. Bosnian Journal of Basic Medical Sciences. 2016;16(3):180-6.

6. AYGUN BK, et al. Posterior reversible encephalopathy syndrome in severe preeclampsia: Case report and literature review. Journal Of The Turkish-German Gynecological Association. 2010;11(4):216-9.

7. BARBOSA AS, et al. Ophthalmic artery-resistive index and evidence of overperfusion-related encephalopathy in severe preeclampsia. Hypertension. 2010;55(1):189-93.

8. BREWER J, et al. Posterior reversible encephalopathy syndrome in 46 of 47 patients with eclampsia. American Journal of Obstetrics and Gynecology [Internet]. 2013;208(6):468.e1-468.e6.

9. CAMARGO EC, et al. Stroke in Pregnancy: An Update. Neurol Clin. 2019;37(1):131-48.

10. CHÁVEZ JAD, CAVALLI RC. Preeclampsia: Vascular pathophysiological mechanism and the basis for early diagnosis and treatment. Revista Brasileira de Ginecologia e Obstetrícia. 2016;38(8):369-72.

11. COZZOLINO M, et al. Therapy and differential diagnosis of posterior reversible encephalopathy syndrome (PRES) during pregnancy and postpartum. Archives of Gynecology and Obstetrics. 2015;292(6):1217-23.

12. DEMIR BC, et al. Comparison of magnesium sulfate and mannitol in treatment of eclamptic women with posterior reversible encephalopathy syndrome. Archives of Gynecology and Obstetrics. 2012;286(2):287-93.

13. DONG X, NAO J. Influential factors and clinical significance of an atypical presentation of posterior reversible encephalopathy syndrome in patients with eclampsia. Neurological Sciences. 2019;40(2):377-84.

14. FINOCCHI V, et al. Magnetic resonance imaging in Posterior Reversible Encephalopathy Syndrome: Report of three cases and review of literature. Archives of Gynecology and Obstetrics. 2005;271(1):79-85.

15. FITTRO K, DIZON R. Understanding posterior reversible encephalopathy syndrome. JAAPA : Official Journal of the American Academy of Physician Assistants. 2018;31(7):31-4.

16. HAMMER ES, CIPOLLA MJ. Cerebrovascular Dysfunction in Preeclamptic Pregnancies. Current Hypertension Reports. 2015;17(8):64.

17. HINCHEY J, et al. A reversible posterior leukoencephalopathy syndrome. New England Journal of Medicine. 1996;334(8):494-500.

18. HU D, et al. Posterior reversible encephalopathy syndrome complicated with subarachnoid hemorrhage in an eclamptic pregnant patient: case report. BMC Neurology. 2018;18(1):1-5.

19. KARUPPANNASAMY D, et al. Cortical visual loss in posterior reversible encephalopathy syndrome in late postpartum eclampsia: Case series. Indian Journal of Ophthalmology. 2014;62(5):635-8.

20. KATSEVMAN GA, et al. Post-partum posterior reversible encephalopathy syndrome requiring decompressive craniectomy: case report and review of the literature. Acta Neurochirurgica (Wien). 2019;161(2):217-24.

21. KURDOGLU Z, et al. Clinical and perinatal outcomes in eclamptic women with posterior reversible encephalopathy syndrome. Archives of Gynecology and Obstetrics. 2015;292(5):1013-8.

22. LEGRIEL S, et al. Determinants of Recovery from Severe Posterior Reversible Encephalopathy Syndrome. PLoS One. 2012;7(9).

23. LIMAN TG, et al. Clinical and radiological differences in posterior reversible encephalopathy syndrome between patients with preeclampsia-eclampsia and other predisposing diseases. European Journal of Neurology. $2012 ; 19(7): 935-43$. 
24. MATTHYS LA, et al. Delayed postpartum preeclampsia: An experience of 151 cases. American Journal of Obstetrics and Gynecology. 2004;190(5):1464-6.

25. ONDEROGLU LS, et al. Posterior leukoencephalopathy syndrome as a cause of reversible blindness during pregnancy. Journal of Obstetrics and Gynaecology Research. 2007;33(4):539-42.

26. PENG WX, et al. Atypical case of reversible posterior leucoencephalopathy syndrome associated with puerperal HELLP syndrome. Archives of Gynecology and Obstetrics. 2008;278(3):269-71.

27. RAJIV KR, et al. Status epilepticus related to pregnancy: Devising a protocol for use in the intensive care unit. Neurology India. 2018;66(6):1629-33.

28. RAZMARA A, et al. Cerebrovascular Complications of Pregnancy and the Postpartum Period. Current Cardiology Reports. 2014;16(10):532.

29. SCHWARTZ RB, et al. Preeclampsia-eclampsia: clinical and neuroradiographic correlates and insights into the pathogenesis of hypertensive encephalopathy. Radiology. 2000;217(2):371-6.

30. SERVILLO G, et al. Posterior reversible encephalopathy syndrome (PRES) in critically ill obstetric patients. Intensive Care Medicine. 2003;29(12):2323-6.

31. SHANKAR J, BANFIEL J. Posterior Reversible Encephalopathy Syndrome: A Review. Canadian Association of Radiologists Journal. 2017;68(2):147-53.

32. SIBAI BM. Diagnosis, Prevention, and Management of Eclampsia, the American College of Obstetricians and Gynecologist. 2005;105(2):402-10.

33. SINGH S, et al. A rare case of early onset severe preeclampsia with PRES. International Journal of Reproduction, Contraception, Obstetrics and Gynecology. 2018;7(3):1239.

34. TAKAHASHI $\mathrm{H}$, et al. Color vision abnormality as the sole manifestation of posterior reversible encephalopathy due to post-partum HELLP syndrome. Journal of Obstetrics and Gynaecology Research. 2017;43(3):592-4.

35. TRIKHA A, et al. Posterior reversible encephalopathy syndrome. Journal of Obstetric Anaesthesia and Critical Care. 2014;4(2):59.

36. WEN Y, et al. Posterior reversible encephalopathy syndrome in pregnancy: a retrospective series of 36 patients from mainland China. Irish Journal of Medical Science. 2017;186(3):699-705. 